\title{
Nivel cognitivo de las preguntas que formulan las educadoras de párvulos
}

\section{Bernardita Tornero}

Universidad de los Andes (Chile)

btornero@uandes.cl

\section{Alicia Truffello}

Universidad de los Andes (Chile) atruffello@uandes.cl

\section{Antonietta Ramaciotti}

Universidad de los Andes (Chile) aramaciotti@uandes.cl

\section{Francisca Valenzuela}

Universidad de los Andes (Chile) mvalenzuela@uandes.cl

\section{Resumen}

El objetivo principal de este estudio es identificar y clasificar en categorías y subcategorías el nivel cognitivo de las preguntas formuladas por las educadoras de párvulos durante la "hora del cuento". Para ello se confeccionó una pauta basándose en la taxonomía de Bloom adaptada por Anderson, y se observó a 63 educadoras de párvulos de segundo nivel de transición provenientes de 37 escuelas particulares subvencionadas de 7 comunas de la región metropolitana de Santiago de Chile. Los resultados señalan que, en promedio, las educadoras formulan 33 preguntas durante "la hora del cuento", pero que a pesar de que la mayoría son preguntas abiertas, demandan bajos niveles cognitivos.

\section{Palabras clave}

Educación inicial, desarrollo cognitivo, pregunta pedagógica, desarrollo del pensamiento, Santiago de Chile (Fuente: Tesauro de la Unesco).

Recepción: 2014-11-14 | Envío a pares: 2015-02-21 | Aceptación por pares: 2015-05-08 | Aprobación: 2015-08-02 


\title{
The Cognitive Level of Questions Asked by Preschool Teachers
}

\begin{abstract}
The primary objective of this study was to identify and classify into categories and subcategories the cognitive level of the questions asked by preschool teachers during "story time". A model based on Bloom's taxonomy, as adapted by Anderson, was constructed for this purpose, and 63 preschool teachers (transition level two) from 37 subsidized private schools in seven communes in the metropolitan area of Santiago de Chile were observed. The results showed that teachers asked 33 questions, on average, during "story time". Even though most are open questions, they demand low levels of cognitive behavior.
\end{abstract}

\section{Keywords}

Early childhood education, cognitive development, pedagogical question, development of thinking, Santiago de Chile (Source: Unesco Thesaurus). 


\title{
Nivel cognitivo das perguntas formuladas pelas educadoras de párvulo
}

\author{
Resumo
}

O objetivo principal deste estudo é identificar e classificar em categorias e subcategorias o nível cognitivo das perguntas formuladas pelas educadoras de criança durante a "hora do conto". Para isso, elaborou-se um documento baseado na taxonomia de Bloom, adaptada por Anderson, e observaram-se 63 educadoras de criança de segundo nivel de transição provenientes de 37 escolas particulares subvencionadas de sete comunidades da região metropolitana de Santiago do Chile. Os resultados indicam que, em média, as educadoras formulam 33 perguntas durante a "hora do conto", mas que, embora a maioria sejam perguntas abertas, demandam baixos niveis cognitivos.

\section{Palavras chave}

Educação inicial, desenvolvimento cognitivo, pergunta pedagógica, desenvolvimento do pensamento, Santiago do Chile. (Fonte: Tesauro da Unesco). 


\section{Introducción}

La pregunta es una de las herramientas pedagógicas más frecuentemente utilizada al interior de la sala de clases. Ella no solo ayuda a evaluar o monitorizar el progreso de los estudiantes y los niveles de comprensión alcanzados por ellos, sino que también permite y promueve distintos niveles de procesamiento de la información. Por esta razón, es importante que los educadores tomen conciencia del rol que la pregunta pedagógica cumple en el proceso de enseñanza-aprendizaje de manera que no sea subutilizada en el aula.

De esta forma, la realización de una pregunta no es un fin en sí mismo, sino un medio de aprendizaje siempre y cuando los educadores sepan qué promueve una pregunta, y cómo y en qué momento se debe formular. Sin embargo, aún hay muchos aspectos de ella que se deben conocer y comprender, a fin de utilizarla para facilitar y potenciar el proceso de aprendizaje de los estudiantes.

Existe numerosa literatura y evidencia empírica a nivel internacional que muestra la relación entre el uso del lenguaje que hacen los educadores, siendo la pregunta una manifestación de este, y el desarrollo de habilidades de pensamiento. Por esta razón, este estudio elaboró una taxonomía que permitió clasificar en distintos niveles de demanda cognitiva las preguntas realizadas por educadoras de párvulos de segundo nivel de transición antes, durante y después de la hora del cuento, en 37 escuelas de la región metropolitana de Santiago de Chile.

En consecuencia, los objetivos de este estudio están orientados a identificar y clasificar en categorías y subcategorías el nivel cognitivo de las preguntas formuladas por las educadoras de párvulos en la hora del cuento. Asimismo, se describió la relación existente entre la frecuencia de las distintas categorías y subcategorías y los momentos de la hora del cuento, y el tipo de pregunta formulada (abierta y cerrada). Finalmente, se analizó la cantidad de preguntas cerradas que eran seguidas por otra pregunta complementaria y se identificó a qué categoría y subcategoría correspondían.

\section{La importancia de la pregunta como herramienta pedagógica}

Vigotsky (1986) fue uno de los primeros autores en describir la relación recíproca que existe entre los procesos de desarrollo del lenguaje y del pensamiento. Su teoría establece que ambas funciones, esencialmente humanas, están mediadas y determinadas por la experiencia que el niño tiene en su contexto social y cultural. Así, a pesar de que ambas funciones tienen raíces genéticas diferentes, llega un momento en que el desarrollo de ellas coincide, produciéndose la verbalización del pensamiento y la racionalización del lenguaje.

Diversos autores (Banyard, Cassells, Green, Hartland, Hayes et al., 1995; Bermeosolo, 2004), a partir de sus estudios en torno a la interacción entre lenguaje y pensamiento, han señalado que los límites de los procesos de pensamiento están determinados por las estructuras lingüísticas del niño. Por esta razón, Craig (2001) sostiene que los procesos lingüísticos cumplen una función de facilitación u obstaculización de los procesos cognitivos, ya que el desarrollo de estos últimos se encuentra íntimamente determinado por los primeros.

Los niños en edad preescolar (como los participantes de este estudio) se encuentran, según Piaget, en la etapa del pensamiento preoperacional. Durante este periodo mejora la representación interna de los sucesos y, de esta manera, se vuelven más independientes de la actividad sensorio-motriz para así comprender lo que forma parte de su entorno (Feldman, 2007).

De acuerdo con Papalia, Wendkos y Duskin (2009), uno de los logros más relevantes de esta etapa es el desarrollo del pensamiento simbólico y, con él, una creciente comprensión de las causalidades y la capacidad de categorización. De esta forma, el niño va desde un aprendizaje ligado a los esquemas 
motores y las percepciones más cercanas del entorno, hacia las representaciones mentales de aquellos elementos que no se encuentran físicamente presentes, pero que han sido observadas en un pasado, para alcanzar finalmente el aprendizaje simbólico.

A pesar de que la centración del pensamiento propio de esta etapa no le permite al niño considerar varios puntos de vista a la vez o tomar en consideración el punto de vista de los demás (Feldman, 2007), su naturaleza intuitiva aumenta su curiosidad y lo lleva a buscar respuestas a las diversas interrogantes mediante el uso del "¿por qué?" (Papalia et al., 2009). Paralelo a esto, el niño cree saber la respuesta a todo tipo de preguntas, sin embargo, no es capaz de explicar cómo lo sabe debido a que tiene una baja base lógica para comprender cómo funciona el mundo. De esta forma, en esta etapa la pregunta es el mecanismo que utiliza el niño para saber que hay una explicación o una respuesta a su por qué, permitiéndole así organizar su realidad (Monfort y Juárez, 2008).

Todas estas actividades, primero motrices y luego verbales, permiten que el niño vaya modificando sus esquemas cognitivos, pasando por procesos de asimilación y acomodación, hasta construir nuevos conocimientos. Es así como al momento de interactuar con el medio y resolver situaciones cotidianas, el niño despliega las herramientas lógicas que posee, logrando desarrollar su pensamiento y construir un nuevo aprendizaje. En este sentido, en el proceso de desarrollo cognitivo del niño, el lenguaje y la pregunta como expresión de este juegan un rol fundamental al ser tareas eminentemente simbólicas que contribuyen al logro de la capacidad de elaborar representaciones mentales y, en consecuencia, desarrollan el pensamiento de los estudiantes (Papalia et al., 2009).

De esta manera, el reconocer la interacción e influencia que existe entre los procesos lingüísticos y los procesos de pensamiento tiene relevantes implicancias para el proceso educativo, ya que trans- forma al lenguaje en la principal y más importante herramienta pedagógica. En el contexto educativo chileno, la pregunta cobra especial relevancia ya que es la estrategia pedagógica más frecuentemente utilizada por los docentes en el aula de clases (Collante, 2010). Ella es la herramienta lingüística por excelencia que permite la interacción entre pares y entre estudiantes-educadores. Por esta razón, Thuel (1995) resalta la importancia de poner atención a la construcción de preguntas, de modo que ellas favorezcan en los estudiantes hacer complejas sus estructuras de pensamiento, que son socializadas a través del lenguaje. De esta forma, la pregunta se constituye como una estrategia educativa pedagógica muy efectiva a la hora de promover el diálogo, la discusión y la reflexión en el aula (Candelas, 2011), y en un medio para impulsar el desarrollo cognitivo de los estudiantes.

Si además se toma en consideración que uno de los factores más influyentes a la hora de determinar si un profesor es efectivo, es el grado de reflexión y cuestionamiento que genera en sus estudiantes (Arancibia, Herrera, Strasser, 2011), la pregunta cobra aún mayor relevancia como herramienta que permite promover niveles desafiantes de reflexión y cuestionamiento en el aula. Una pregunta oportuna puede abrir un universo de posibles respuestas que enriquecen el pensamiento con soluciones creativas a los problemas, y abre una multiplicidad de caminos que a veces ni siquiera el docente es capaz de anticipar. Sin embargo, si esta no se utiliza intencionadamente con objetivos claros de aprendizaje, no se estaría a provechando el potencial que ella tiene.

De esta forma, debido a que la pregunta es una estrategia pedagógica eficaz para promover el desarrollo y fortalecimiento de las estructuras cognitivas de los estudiantes (Polanco, 2004), varios autores (González García, 2007; Polanco, 2004) señalan que las instancias de formación docente debieran mostrar a los educadores la importancia del rol que esta juega como herramienta de aprendizaje y, al 
mismo tiempo, entregarles a ellos las herramientas necesarias para que realicen preguntas de calidad en el aula, que estimulen procesos cognitivos complejos y diversos en los estudiantes. De lo anterior se desprende, que la correcta utilización de la pregunta como herramienta pedagógica requiere no solo de un proceso de aprendizaje previo, sino además de una minuciosa planificación y evaluación de las preguntas que los educadores pretenden utilizar en momentos particulares del proceso educativo, de manera que cumplan un rol significativo al interior de este (Burton y Habenicht, 2004).

\section{Las preguntas que realmente impactan en el aula}

La sección anterior permitió establecer las relaciones teóricas que existen entre la utilización de preguntas y la promoción de procesos de pensamiento. Sin embargo, para que esta relación sea tal, es necesario determinar qué tipo de preguntas son las que efectivamente promueven procesos cognitivos superiores. A continuación, se presentará una clasificación de los distintos tipos de preguntas utilizadas por los educadores a fin de establecer cuáles son más deseables en términos del impacto que generan en la promoción de habilidades de pensamiento.

Una de las formas más tradicionales para clasificar el tipo de preguntas pedagógicas es la que las agrupa en cerradas o abiertas. Las preguntas cerradas son aquellas que generalmente tienen un número limitado de respuestas posibles (por ejemplo, un color; o "si" y "no") o bien implican seleccionar entre varias alternativas presentadas en la pregunta misma (Wasik, 2010). Este tipo de preguntas requiere respuestas muy cortas y predeterminadas por el educador, quien la mayoría de las veces sabe exactamente lo que los estudiantes deben responder y espera una respuesta especifica (de Rivera, Girolametto, Greenberg y Weitzman, 2005). Debido a que estas preguntas generalmente cuentan con una única respuesta correcta y no requieren procesos de elaboración más profundos ni el uso de información nueva por parte de los estudiantes, usualmente se intenta limitar su aparición y su presencia durante el proceso educativo (Monfort y Juárez, 2008).

Por su parte, de acuerdo con la literatura, las preguntas abiertas demandan mayores grados de elaboración y abstracción de parte de los estudiantes, ya que generalmente implican establecer relaciones entre distintos aspectos de un enunciado, emitir un juicio o una opinión basándose en información con la que ya se cuenta, activar conocimientos previos, entre otros (De Rivera et al., 2005). Debido a las características de este tipo de preguntas, el educador usualmente no es capaz de prever el desenlace que estas van a tener ni el curso que va a tomar la conversación, ya que este depende eminentemente de las intervenciones que hagan sus estudiantes en el transcurso de la misma.

Respecto a la deseabilidad y frecuencia de las preguntas de tipo abierto en comparación con las cerradas, Honig y Wittmer (1982) señalan que los educadores debieran favorecer el uso de preguntas abiertas, ya que estas generalmente estimulan respuestas más largas y elaboradas de parte de los estudiantes y, además, tienen un mayor potencial de promover respuestas con mayores grados de complejidad tanto desde un punto de vista cognitivo como lingüístico (De Rivera et al., 2005).

Otra forma muy frecuentemente utilizada para clasificar las preguntas es la tipología que las agrupa en convergentes o divergentes dependiendo del tipo de procesos de pensamiento que estas favorecen. Las preguntas convergentes, como su nombre lo indica, son aquellas que frente a la resolución de un problema promueven soluciones tradicionales. Esto quiere decir que requieren de la aplicación de un proceso ya existente o predeterminado como respuesta (Álvarez, 2010). Por esta razón, diversos autores (Villén, 2009; Abarca, Sala y Marzo, 2002) las identifican con las preguntas cerradas, ya que ambos tipos de interrogantes tienen un universo bastante limitado de respuestas. 
Por su parte, las preguntas divergentes demandan procesos de razonamiento inéditos de parte de los estudiantes, dado que no están determinadas por un proceso rígido ni único, dejando abiertas una multiplicidad de opciones de respuesta. Por esta razón, este tipo de preguntas fomenta el desarrollo de la capacidad creativa y reflexiva en la sala de clases (Álvarez, 2010; Villén, 2009; Abarca et al., 2002). Estas preguntas tienden a asemejarse a las abiertas tanto por sus características constitutivas como por el tipo de respuestas que promueven.

Aunque, en términos generales, la terminología anterior hace referencia a dos grandes categorías de preguntas en función del tipo de respuestas que estas suscitan, es difícil afirmar que la diversidad de preguntas se agota en dichas categorías. Por esta razón, a continuación se presenta la tipología sugerida por Huck (1976), que da cuenta de mejor manera de la multiplicidad y complejidad de las preguntas que se realizan en el aula. Los autores mencionan categorías distintas en función de los niveles de pensamiento que las preguntas demandan y, en consecuencia, del grado de abstracción asociado a ellas. Con un grado de abstracción creciente, las siete categorías elaboradas son las siguientes: memoria, comprensión, interpretación, aplicación, análisis, sintesis y evaluación.

Es posible observar que, más allá de las diferencias observadas en las tres tipologías señaladas, todas ellas implican un reconocimiento de que los niveles de abstracción requeridos por las preguntas elaboradas son los que realmente marcan la diferencia en la complejidad de los procesos cognitivos activados en los estudiantes. De esta forma, del nivel de abstracción que tenga que realizar un estudiante al contestar una pregunta depende cuánta reflexión requiere su respuesta, qué nivel de pensamiento realiza para contestarla, o qué tipo de proceso cognitivo debe poner en marcha para hacerlo. Es así como a pesar de que los distintos tipos de preguntas son necesarias y cumplen una función dentro de los diferentes momentos del proceso de enseñanza- aprendizaje, aquellas que demandan niveles más altos de abstracción son las que realmente desafían las estructuras cognitivas de los estudiantes y promueven el desarrollo del pensamiento de estos.

\section{Evidencia respecto de la capacidad de los docentes de elaborar preguntas de alto nivel cognitivo}

No obstante la evidencia existente que indica la importancia de la pregunta como herramienta pedagógica orientada a promover procesos cognitivos complejos en los estudiantes, es posible afirmar que esta sigue estando subutilizada en el aula, no por el hecho de que los profesores no la utilicen como herramienta, porque de hecho, como se indicó, es uno de los medios más utilizado para generar interacción, sino más bien porque no se ha explorado de manera sistemática todo su potencial en el desarrollo de habilidades de pensamiento complejas.

En este contexto, Polanco (2004) menciona que gran parte de los educadores no solo desconoce la importancia del rol que la pregunta desempeña en el desarrollo cognitivo de sus estudiantes, sino que además tiende a elaborar preguntas mayoritariamente de carácter convergente, que no estimulan procesos reflexivos y creativos en los estudiantes. Es esta misma línea, Wells (2002) señala que en el discurso de los educadores predominan las preguntas cerradas, que tienen solo una solución correcta, por lo que los esfuerzos de los estudiantes están dirigidos a encontrar dicha respuesta y no a pensar sobre ella. Como resultado, la monitorización de los educadores se orienta a evaluar la correspondencia entre sus preguntas y las respuestas cortas de los estudiantes. Este tipo de patrones instruccionales e interaccionales son completamente opuestos a aquellos contextos de aprendizajes en los que se logra un diálogo multimodal, donde el principal objetivo es alcanzar la comprensión colectiva de los tópicos que se discuten (Smith, Hardman, Wall y Mroz, 2004).

En esta misma línea, Zucker, Justice, Piasta y Kaderavek (2010) llevaron a cabo un estudio orien- 
tado a investigar el tipo de preguntas que los educadores realizan durante la lectura compartida y a establecer si las habilidades lingüísticas que estas demandaban eran de carácter literal o inferencial, siendo estas últimas de un grado mayor de complejidad por el nivel de abstracción que requieren. Los resultados son consistentes con la evidencia presentada anteriormente, ya que sugieren que los educadores realizan preguntas de tipo literal de manera más frecuente que preguntas de tipo inferencial.

En este contexto, Moses (2005) afirma que es preocupante la clase de preguntas que las educadoras usualmente formulan a sus estudiantes, ya que estos tienden a dar respuestas de tipo "si" o "no" pero no porque no sean capaces de elaborar respuestas más complejas, sino, sobre todo, por la naturaleza y las características de las preguntas formuladas. Este hecho es especialmente problemático si se considera que la evidencia señala que el desarrollo cognitivo de los estudiantes está fuertemente relacionado con el tipo de preguntas planteadas en la sala de clases y con los procesos de pensamiento que dichas preguntas activan. En consecuencia, los resultados implicarían que los niveles de razonamiento que los estudiantes realizan al interior del aula son de carácter eminentemente literal, en desmedro del inferencial, lo que tiene consecuencias negativas en sus posibilidades de desarrollo cognitivo.

Por esta razón, diversos autores (Doyle y Carter, 1996; Holt-Reynolds, 1992; Kagan, 1992) han señalado que una de las principales razones que explica la falta de desarrollo de habilidades de pensamiento en los estudiantes en el sistema educativo es la dificultad que experimentan los educadores en elaborar un discurso pedagógico con fuertes componentes mentales, que es justamente una potente herramienta pedagógica para promover el desarrollo cognitivo. Como resultado, los educadores se dirigen a los estudiantes principalmente para dar instrucciones o explicar un contenido.

Esta conclusión fue corroborada por la evidencia proporcionada por un estudio realizado en siete países (Australia, República Checa, Hong Kong, Japón, Holanda, Suiza y Estados Unidos), que mostró que en todos ellos los educadores tendían a usar la pregunta como una manera de abordar un concepto más que como una estrategia para explicar o analizar un proceso (Hiebert et al., 2003). En consecuencia, las preguntas que los educadores usualmente realizan requieren de los estudiantes procesos como describir o clasificar, que no demandan procesos superiores de pensamiento como son analizar, deducir, hacer hipótesis, inferir, evaluar, etc.

El caso de Chile no es la excepción a la tendencia observada internacionalmente. Un estudio realizado por González, Preiss y San Martín (2008) analizó el desempeño de los educadores al interior del aula con el fin de evaluar hasta qué punto el discurso elaborado por ellos estaba orientado a promover el desarrollo de habilidades de pensamiento de orden superior. El equipo de investigación encontró que, en general, las preguntas y el discurso de los educadores estaba principalmente orientado a controlar y modelar el comportamiento de los estudiantes o a corroborar información más que a desarrollar procesos cognitivos complejos.

Asimismo, otro estudio realizado por Salazar y Márquez (2012) mostró que los educadores generalmente no estimulaban la inferencia y la formulación de hipótesis, que había una escasa resolución de problemas como estrategia de inicio y activación de aprendizajes previos, que no promocionaban lo suficiente el pensamiento críticoanalítico y que no desafiaban el pensamiento, lo que en la práctica generaba que los estudiantes no le dieran significado al aprendizaje. Respecto a este hecho, Zuleta (2005) señala que en el ámbito educativo chileno siempre ha prevalecido una cultura de sobrevalorar la importancia de la respuesta por sobre la pregunta, ya que los educadores tienen puesto el énfasis en evaluar la correspondencia de las respuestas entregadas por los estudiantes sin ser conscientes de que lo verdaderamente importante es realizar buenas preguntas que sean capaces de motivar pensamientos de orden más complejo. 
Toda esta evidencia es especialmente preocupante si se considera no solo que los educadores tienden a no realizar preguntas demandantes cognitivamente a los estudiantes, sino también que son ellos los que elaboran la mayor parte del discurso que tiene lugar en el aula (Childs y McNicholl, 2007; McLoughlina y Mynard, 2009). De hecho, el estudio realizado en los siete países mencionados con anterioridad, concluyó que en todos ellos los educadores hablaban muchísimo más que los estudiantes, en una proporción de al menos 8 a 1 (Hiebert et al., 2003), lo que sugiere que los estudiantes no tienen el espacio de usar su propio lenguaje y el de sus compañeros como una oportunidad de aprendizaje y de desarrollo cognitivo. De esta forma, el minimizar los espacios de interacción y participación de los estudiantes al interior de la sala de clases tiene efectos nocivos en su proceso de aprendizaje, ya que como dicen Eyzaguirre y Fontaine (2008), "el manejo de clases, entendido como las conductas del profesor destinadas a minimizar las interrupciones y maximizar las posibilidades de que las interacciones en la clase se orienten al aprendizaje, es una de las variables del profesor que mayor influencia tienen para lograr éxitos en el aprendizaje" (p. 173).

\section{La hora del cuento: contexto ideal para la formulación de preguntas}

Como se ha señalado hasta ahora, la interacción verbal y el discurso que se desarrolla en el aula tiene un poderoso potencial de estimulación cognitiva (Bermeosolo, 2004). De esta forma, el rol que tiene el educador durante el transcurso de la conversación que mantiene con los estudiantes, cobra vital importancia como contexto para promover el desarrollo del pensamiento.

En este sentido, leer un cuento puede ser una instancia de establecimiento de diálogo entre el educador y los estudiantes que facilite el formular preguntas de alto nivel, que permitan favorecer en los estudiantes procesos cognitivos superiores. Esta debiera ser una actividad diaria, cuidadosamente pla- nificada (Otto, 2008), de manera que contribuya favorecer el pensamiento de los niños (Wiseman, 2011).

Entendiendo el cuento como un relato breve, de hechos imaginarios, con un desenlace argumental sencillo cuya finalidad puede ser moral o recreativa y que estimula la imaginación del niño (Rodari, 2008), se consideró en esta investigación como hora del cuento, aquella actividad de aprendizaje en que el profesor lee o narra un cuento a sus alumnos, con las características propias de la forma de lectura que se escojan (en voz alta, compartida, dialógica, entre otras). Se basa en la propuesta realizada por Gabriela Mistral, quien la describió como una iniciativa para ser desarrollada en la escuela, por el profesor o el bibliotecario, al interior de la sala de clases, y que busca favorecer el gusto por la lectura. De acuerdo con lo señalado por la autora, la actividad debe terminar en una conversación con los niños en torno a lo escuchado, con la intención de despertar el interés por aprender y favorecer la reflexión (Edwards, 2008). Es en este último aspecto (conversación) en el que se encuentra el sentido pedagógico de la actividad. La selección del material literario queda a criterio del profesional, pero debe considerar el grupo de destinatarios, las características del texto, los objetivos establecidos para la sesión y las características del mediador, sus gustos y habilidades (Beuchat et al., 2013).

El Ministerio de Educación de Chile (2010) afirma que en este momento de la rutina pedagógica, es indispensable la formulación de preguntas que tengan como objetivo formular inferencias, realizar predicciones, conceptualizar, ampliar el vocabulario, crear imágenes mentales, confirmar o rechazar hipótesis, plantear preguntas y responderlas. Berríos et al. (2010) agregarían que este espacio educativo debe durar a lo menos quince minutos, y en él deben trabajarse distintos aspectos del desarrollo del lenguaje, ya sea oral o escrito. A través de la interacción comunicativa que se da en esta práctica pedagógica, el adulto es capaz de realizar preguntas con el objetivo de hacer al niño reflexionar y razonar sobre lo 
escuchado, potenciando así el desarrollo cognitivo y el desarrollo del pensamiento.

Entre las ventajas de la lectura de textos literarios en voz alta se han mencionado la aceleración de la expansión del vocabulario de los estudiantes, la promoción de sus habilidades de comprensión oral (Dickinson y Smith, 1994) y todos aquellos procesos complementarios a la alfabetización inicial (Justice y Pentimonti, 2010; Navarro, 2000). Las características propias de la hora del cuento y de la rutina de elaboración de preguntas asociada a ella, lo transforman en un momento ideal para elaborar preguntas de calidad que permitan a los estudiantes desarrollar procesos de pensamiento de nivel superior. De esta forma, la hora del cuento construye las bases necesarias para el aprendizaje futuro de los estudiantes.

A través de la interacción comunicativa, el educador realiza preguntas con el objetivo de hacer a los estudiantes reflexionar y razonar sobre lo escuchado, potenciando así el desarrollo cognitivo y de su pensamiento. Sin embargo, para que realmente las preguntas se constituyan en promotoras del pensamiento y de procesos cognitivos superiores durante la hora del cuento, estas tienen que ser planificadas de antemano con una causa y finalidad, ya que de esta manera se puede conducir el diálogo a una instancia donde se desarrolle el pensamiento reflexivo de los niños y su creatividad a través de preguntas divergentes y abiertas (Polanco, 2004).

La hora del cuento contempla tres momentos importantes y distintivos; antes, durante y después (Dickinson y Smith, 1994), y cada uno de ellos se convierte en una instancia única para proponer preguntas que apunten al desarrollo de habilidades cognitivas diferentes.

El "antes" se refiere al periodo en el que el docente muestra el libro a los estudiantes y plantea preguntas respecto de él. De acuerdo con Berríos et al. (2010), el "antes" es una buena instancia para activar los conocimientos previos de los estudiantes, a través de la exploración de sus experiencias y creencias relacionadas con el tema por tratar. Sugiere además promover en los alumnos la realización de predicciones antes de leer el texto y el desarrollo de inferencias respecto al tipo de texto que se leerá, de acuerdo con diversas claves de diagramación u otras claves lingüísticas.

El "durante" es el momento en que se realiza la lectura, en donde también se pueden hacer predicciones sobre lo que no ha sucedido aún en la historia, y relacionarlo con lo que ya se ha leído (Castro y Rodríguez, 2005). En este momento del cuento es importante trabajar el vocabulario, promoviendo la deducción de los significados de las palabras que no se conocen a partir del contexto. Además, el educador puede aclarar dudas acerca del texto y así asegurarse de que los niños están comprendiendo el cuento.

Por último, el "después" de la lectura se relaciona con las actividades realizadas una vez terminado el cuento (Dickinson y Smith, 1994) y es un momento ideal para sintetizar las ideas principales de la historia, elaborar un pequeño resumen de los hechos, y formular variadas preguntas acerca de lo leído (Castro y Rodríguez, 2005). Ibarra (2000) señala, además, que después de leer, es muy importante realizar preguntas que favorezcan la transferencia y la metacognición.

\section{Metodología}

\section{Diseño de investigación}

El diseño de investigación fue exploratorio, de carácter descriptivo, ya que el objetivo del estudio estaba justamente enfocado a describir el tipo de preguntas que las educadoras de párvulos de segundo nivel de transición utilizan antes, durante y después de la hora del cuento en establecimientos particulares subvencionados de la región metropolitana de Santiago de Chile. De acuerdo con Cohen, Manion y Morrison (2007), este tipo de diseño es es- 
pecialmente adecuado cuando no existe mayor información respecto del fenómeno en estudio, como es el caso de esta investigación.

Por otra parte, el presente estudio fue de tipo cuantitativo (Hernández, Fernández y Baptista, 2010), ya que se realizaron conteos de frecuencia de la cantidad y tipo de preguntas elaboradas por las educadoras de párvulos de segundo nivel de transición en cada uno de los tres momentos de la hora del cuento.

\section{Selección y características de la muestra}

El tipo de muestreo utilizado fue intencionado por conglomerado, donde la unidad más pequeña para ser seleccionada fueron comunas de la región metropolitana de la ciudad de Santiago de Chile y no los establecimientos educacionales de manera individual. La justificación para la utilización de este tipo de muestreo fue netamente de factibilidad, ya que debido a que las comunas son administraciones territoriales que agrupan establecimientos educacionales y estas debían ser abordadas por el equipo de investigación, era razonable pensar que las escuelas incluidas en el estudio no podían estar tan distantes unas de otras a fin de poder incluir a un mayor número de ellas.

Como resultado de este procedimiento de muestreo, siete fueron las comunas que participaron en el estudio: San Miguel, La Reina, Ñuñoa, Peñalolén, Macul, San Joaquín y Providencia. Para elegirlas, se consideraron criterios de similitud económica, educativa y social (tabla 1). De esta manera, fue posible concentrar a todas las escuelas en un solo grupo, ya que el estudio no intentaba buscar diferencias entre los distintos tipos de ellas.

Dentro de las siete comunas seleccionadas, se invitaron a participar a todas escuelas de dependencia particular subvencionada, por ser el tipo de escuelas al que asiste el $54 \%$ de los estudiantes chilenos (Instituto Nacional de Estadísticas, Gobierno de Chile, 2011). El 64,9\% de las 57 escuelas contactadas accedió a participar en el estudio (tabla 2) dando un total de 37 escuelas participantes.

Tabla 1. Caracterización de las comunas participantes

\begin{tabular}{|l|c|}
\hline \multicolumn{1}{|c|}{ Indicador } & Característica \\
\hline Nivel Socioeconómico & C2 \\
\hline Ingreso mensual familiar promedio & $\begin{array}{c}\$ 1.000 .000 \\
\text { (USD 1.669) }\end{array}$ \\
\hline Nivel educativo promedio & $\begin{array}{c}\text { Escolaridad } \\
\text { completa }\end{array}$ \\
\hline No pobreza promedio (\%) & 89,06 \\
\hline Mujeres jefas de hogar promedio (\%) & 45,26 \\
\hline Analfabetismo Promedio (\%) & 2,56 \\
\hline
\end{tabular}

Tabla 2. Cantidad de establecimientos participantes por comuna

\begin{tabular}{|c|c|}
\hline Nombre comuna & No. de establecimientos \\
\hline Educacionales & \\
\hline San Miguel & 9 \\
\hline La Reina & 3 \\
\hline Ñũoa & 4 \\
\hline Peñalolén & 3 \\
\hline Macul & 6 \\
\hline San Joaquín & 11 \\
\hline Providencia & 1 \\
\hline Total & 37 \\
\hline
\end{tabular}

Luego de que las escuelas manifestaron su interés en participar en el estudio, se extendió la invitación a las educadoras de párvulos de segundo nivel de transición de las mismas; 63 accedieron a que sus salas de clases fueran observadas durante la hora del cuento. La mayoría de las salas observadas tenían una composición mixta, es decir, estaban conformadas tanto por niños como por niñas (tabla 3). Sin embargo, más de la mitad de ellas tenían una mayor proporción de niños (tabla 4). Finalmente, el promedio de estudiantes por sala era de 23,16 donde 75,86\% de ellas tenía entre 11 y 30 estudiantes (tabla 5). 
Tabla 3. Composición de las salas observadas según género

\begin{tabular}{|l|c|}
\hline \multicolumn{1}{|c|}{ Tipos de salas de clases } & Salas (\%) \\
\hline Solo niñas & 1,72 \\
\hline Mixtas & 86,21 \\
\hline Solo niños & 12,07 \\
\hline
\end{tabular}

Tabla 4. Proporción de niños y niñas por sala

\begin{tabular}{|c|c|}
\hline $\begin{array}{c}\text { Porcentaje de niños del } \\
\text { total de estudiantes }\end{array}$ & $\begin{array}{c}\text { Salas } \\
\text { (\%) }\end{array}$ \\
\hline $0-49$ & 34,88 \\
\hline 50 & 11,63 \\
\hline $51-100$ & 53,49 \\
\hline
\end{tabular}

Tabla 5. Cantidad total de estudiantes por sala

\begin{tabular}{|c|c|}
\hline $\begin{array}{c}\text { Rango de } \mathbf{n}^{\circ} \text { de } \\
\text { estudiantes }\end{array}$ & Salas (\%) \\
\hline $0-10$ & 6,90 \\
\hline $11-20$ & 31,03 \\
\hline $21-30$ & 44,83 \\
\hline $31-40$ & 17,24 \\
\hline
\end{tabular}

\section{Métodos de recolección y análisis de datos}

El rol del equipo de investigación fue de observador. De acuerdo con DeWalt y DeWalt (2002), la observación es una estrategia de investigación que permite comprender el contexto natural de los participantes a través de observar las actividades y los procesos que tienen lugar espontáneamente en el campo de estudio. Dichas observaciones estuvieron guiadas y fueron registradas en una pauta de observación confeccionada especialmente para los propósitos de este estudio, basándose en la clasificación de los momentos de observación de la hora del cuento desarrollada por Dickinson y Smith (1994). Ellos dividen la hora del cuento en tres momentos principales: antes, durante y después de la lectura, a fin de identificar en qué momento del proceso las educadoras realizan las preguntas a sus estudiantes.
Con el objeto de asegurar la validez de las observaciones registradas en dicha pauta, cada observación fue conducida por un integrante del equipo de investigación y, adicionalmente, grabada en audio utilizando una grabadora portátil de voz, para poder cotejar que las preguntas registradas en cada uno de los momentos fueran un reflejo fidedigno de lo que efectivamente había ocurrido durante la hora del cuento. Cada una de las 63 educadoras fue observada en dos oportunidades.

\section{Categorías de análisis}

Para la descripción y categorización de las preguntas registradas durante la hora del cuento, se utilizó una tabla de categorías basada en la Taxonomía de los Objetivos de la Educación elaborada por Bloom en 1956 y posteriormente revisada por Anderson y Krathwohl (2001). La taxonomía original de Bloom agrupaba los objetivos educacionales en seis categorías, ordenadas según un continuo: conocimiento, comprensión, aplicación, análisis, síntesis y evaluación (Bloom et al., 1990). Años más tarde, Anderson y Krathwohl (2001) realizaron una revisión de la taxonomía original incorporando los resultados de la investigación educativa más actual. Como resultado, ordenaron las categorías y sus procesos cognitivos relacionados de menor a mayor complejidad.

De esta forma, basándose en la taxonomía de Bloom revisada por Anderson y Krathwohl (2001), el equipo de investigación adaptó dicha taxonomía para los propósitos del estudio. Con base en dicha adaptación, se confeccionó una pauta de clasificación para categorizar las preguntas elaboradas por las educadoras de párvulos de segundo nivel de transición antes, durante y después de la hora del cuento. Es importante mencionar que, si bien las categorías sí se encuentran en un orden de la más simple a la más compleja, las subcategorías dentro de cada categoría no se encuentran ordenadas de esta manera. Además de las categorías, cada pregunta tenía un código que indicaba si era una pregunta abierta o cerrada, y si se había realizado antes, durante o 
después de la lectura del cuento (tablas 6 y 7). Varias de las subcategorías identificadas son producto de un proceso de pilotaje en las que se conservaron aquellas que fueron emergiendo en el proceso de codificación.

\section{Tabla 6. Clasificación de las preguntas según tipo y momento}

\begin{tabular}{|c|c|}
\hline Tipo de pregunta & Código \\
\hline Cerrada & 0 \\
\hline Abierta & 1 \\
\hline Momento & Código \\
\hline Antes & 1 \\
\hline Durante & 2 \\
\hline Después & 3 \\
\hline
\end{tabular}

Respecto al análisis de los datos recolectados, se realizaron conteos de frecuencia orientados a describir los distintos tipos de preguntas que las educadoras de párvulos formulan a los estudiantes en los diferentes momentos de la hora del cuento. Para continuar el análisis, se observaron las categorías y subcategorías, ya que se quería explorar además cuántas preguntas de cada categoría y subcategoría se relacionan con cada tipo de pregunta abierta o cerrada. Finalmente, se analizó con qué frecuencia las educadoras formulaban una segunda pregunta (o pregunta complementaria) después de plantear una pregunta cerrada y de qué tipo eran ellas.

En términos de asegurar la confiabilidad interevaluador respecto a la categorización de cada

Tabla 7. Clasificación de las preguntas según nivel de complejidad

\begin{tabular}{|c|c|c|c|}
\hline Categoría & Definición & Subcategoria & Definición \\
\hline \multirow{4}{*}{ Recuperar } & \multirow{4}{*}{$\begin{array}{c}\text { Recordar información } \\
\text { previamente } \\
\text { aprendida }\end{array}$} & Reconocer & Identificar un elemento, objeto, sujeto o suceso \\
\hline & & Reproducir & Volver a producir según lo aprendido, tomando como base lo conocido \\
\hline & & Nombrar & $\begin{array}{l}\text { Mencionar el nombre del cuento, personajes, lugares (palabras } \\
\text { aisladas) }\end{array}$ \\
\hline & & Describir & $\begin{array}{c}\text { Señalar rasgos de algo o alguien, lo que permite identificarlo y } \\
\text { distinguirlo de otros }\end{array}$ \\
\hline \multirow{4}{*}{ Comprender } & \multirow{4}{*}{$\begin{array}{l}\text { Entender, apropiarse } \\
\text { de aquello que se ha } \\
\text { aprendido }\end{array}$} & Exponer & Hablar sobre un tema con las propias palabras \\
\hline & & Secuenciar & Ordenar la aparición de sucesos \\
\hline & & Definir & Señalar el significado de una palabra o concepto \\
\hline & & Sustituir & Cambiar un elemento, un enunciado o situación por otra equivalente. \\
\hline \multirow{3}{*}{ Aplicar } & \multirow{3}{*}{$\begin{array}{l}\text { Emplear o hacer uso } \\
\text { de un conocimiento }\end{array}$} & Ejecutar & Representar o realizar una acción. \\
\hline & & Transferir & Traspasar un conocimiento a una situación nueva similar \\
\hline & & Hacer hipótesis & Predecir resultados para confirmarlos posteriormente \\
\hline \multirow{5}{*}{ Analizar } & \multirow{5}{*}{$\begin{array}{l}\text { Distinguir las } \\
\text { partes de un todo y } \\
\text { relacionarlo con su } \\
\text { estructura global }\end{array}$} & Distinguir & Diferenciar las partes de un todo \\
\hline & & Explicar & Aclarar la causa o motivo de algo \\
\hline & & Comparar & Contrastar aspectos, distinguiendo semejanzas y diferencias entre ellos \\
\hline & & Categorizar & Agrupar a partir de un criterio \\
\hline & & Sintetizar & Seleccionar las ideas principales del tema tratado \\
\hline \multirow{2}{*}{ Evaluar } & \multirow{2}{*}{$\begin{array}{l}\text { Estimar o apreciar } \\
\text { el valor de un sujeto, } \\
\text { objeto o situación }\end{array}$} & Juzgar & $\begin{array}{l}\text { Determinar si el proceso fue bien realizado. Formar opinión sobre algo } \\
\text { o alguien }\end{array}$ \\
\hline & & Justificar & $\begin{array}{c}\text { Argumentar a favor o en contra de un razonamiento, situación o } \\
\text { proceso }\end{array}$ \\
\hline Crear & Producir algo nuevo & Inventar & Proponer algún elemento novedoso \\
\hline
\end{tabular}


ISSN $0123-1294$ | Educ.Educ. Vol. 18. No. 2 | Mayo-Agosto de 2015 | pp. 261-283.

Universidad de La Sabana | Facultad de Educación

una de las preguntas registradas en la pauta de observación, el procedimiento que se utilizó fue que cada investigador-observador de la misma hora del cuento categorizaba de manera individual la lista de preguntas registradas y, solo una vez terminado dicho proceso, dos investigadores comparaban sus respectivas codificaciones a fin de evaluar el grado de coherencia entre ambas. En los casos en que dicha categorización era discordante, se llegaba a un consenso en el equipo de investigación.

\section{Procedimientos éticos}

Como se mencionó en el apartado anterior, el muestro de este estudio fue estratificado, donde la unidad más pequeña seleccionada fueron comunas completas y no establecimientos educacionales ni educadoras de párvulo individuales. De esta manera, este tipo de procedimiento de muestreo tiene el riesgo de incorporar en el estudio individuos que no han sido debidamente informados o que no han dado explícitamente su consentimiento para participar en el estudio.

Para manejar este hecho, de acuerdo con los procedimientos éticos correspondientes, la inclusión de las educadoras de párvulos que finalmente fueron observadas se produjo solo después de que cada una de ellas hubiera firmado un consentimiento informado para ser parte de esta investigación. De esta forma, más allá de que la comuna hubiera sido seleccionada por los procedimientos anteriormente descritos, y sin importar que el establecimiento educacional mismo hubiera manifestado su interés de participar en el estudio, cada educadora de párvulos tenía el derecho de aceptar o rechazar voluntariamente su participación en el mismo. Dicho consentimiento informaba de manera explícita que la aceptación de participar en el estudio implicaba dos observaciones de aula durante la hora del cuento y la grabación de audio de las mismas. Sin embargo, también se les aseguraba a las educadoras que sus identidades y todos los datos recolectados iban a ser manejados bajo estrictos procedimientos de confi- dencialidad, que iban a ser conocidos exclusivamente por el equipo de investigación y que iban a ser utilizados solo para los fines de este estudio.

\section{Resultados}

En términos generales, después de observar dos veces a cada una de las 63 educadoras de párvulos de segundo nivel de transición que participaron en el estudio, fue posible concluir que durante la hora del cuento la cantidad total de preguntas elaboradas fue de 33 en promedio. A continuación se presentarán en detalle los resultados obtenidos de acuerdo con el tipo de preguntas elaboradas y a qué categoría y subcategoría corresponden.

\section{Frecuencia de las preguntas por tipo y momento en que se realizan}

Luego de realizar un conteo de las preguntas abiertas y cerradas que las educadoras de párvulos elaboraban durante la hora del cuento, fue posible observar que las abiertas contaban con la frecuencia más alta (tabla 8).

Tabla 8. Frecuencia de preguntas por tipo

\begin{tabular}{|c|c|}
\hline Momento de la lectura & \% \\
\hline Abierta & 76,35 \\
\hline Cerrada & 23,65 \\
\hline Total & 100 \\
\hline
\end{tabular}

Del mismo modo, si se considera solamente el momento de la hora del cuento en que las educadoras de párvulos realizan la mayor cantidad de preguntas, es posible señalar que la mayor cantidad de ellas, tanto abiertas como cerradas, se concentra después de la lectura (tabla 9).

Tabla 9. Frecuencia de preguntas por momento de la lectura

\begin{tabular}{|c|c|}
\hline Momento de la lectura & $\%$ \\
\hline Antes & 9,42 \\
\hline Durante & 17,62 \\
\hline Después & 72,94 \\
\hline Total & 100 \\
\hline
\end{tabular}


Finalmente, al cruzar la información respecto del tipo de preguntas abiertas o cerradas y el momento de la hora del cuento en que estas son realizadas, es posible señalar que la distribución es bastante homogénea, es decir, no se observan diferencias sustantivas entre el tipo de pregunta abierta o cerrada y el momento de la hora del cuento en que ellas son realizadas (tabla 10). Sin embargo, es importante destacar que el leve aumento en el porcentaje de preguntas cerradas después de la lectura, se puede explicar en la alta frecuencia de la pregunta “¿Les gustó el cuento?", que es cerrada, de la categoría "Evaluar", y es planteada al término del cuento.

Tabla 10. Frecuencia de preguntas por tipo y momento de la lectura

\begin{tabular}{|c|c|c|c|c|}
\hline \multirow{2}{*}{$\begin{array}{c}\text { Tipo de } \\
\text { pregunta }\end{array}$} & \multicolumn{4}{|c|}{$\%$} \\
\cline { 2 - 5 } & Antes & Durante & Después & Total \\
\hline Abierta & 10,62 & 18,40 & 70,97 & 100 \\
\hline Cerrada & 5,53 & 15,12 & 79,33 & 100 \\
\hline
\end{tabular}

\section{Frecuencia de las preguntas por categoría y} subcategoría

A pesar de que las preguntas abiertas fueron las más frecuentemente observadas en los tres momentos de la hora del cuento y, por tanto, se podría pensar que el nivel de demanda cognitiva exigido por ellas sería relativamente alto, al categorizar las preguntas de acuerdo con los seis niveles descritos anteriormente, se encontró que a medida que aumentaban los niveles de demanda cognitiva de las categorías, disminuía la frecuencia de aparición de ese tipo de preguntas (tabla 11). De esta forma, la categoría con mayor frecuencia fue la de recuperar con un $60,20 \%$ y la más baja la de crear con un $0,50 \%$. Con base en esta evidencia, es posible señalar que el tipo de preguntas utilizadas por las educadoras de párvulos de segundo nivel de transición durante la hora del cuento demandan en su mayoría bajos niveles de procesamiento cognitivo de parte de los estudiantes.
Tabla 11. Frecuencia de preguntas abiertas por categoría y subcategoría de análisis

\begin{tabular}{|c|c|c|}
\hline Categoria & Subcategoría & $\%$ \\
\hline \multirow{5}{*}{ Recuperar } & & 60,20 \\
\hline & Reconocer & 36,20 \\
\hline & Reproducir & 1,60 \\
\hline & Nombrar & 13,1 \\
\hline & Describir & 9,30 \\
\hline \multirow{5}{*}{ Comprender } & & 12,8 \\
\hline & Exponer & 6,10 \\
\hline & Secuenciar & 3,80 \\
\hline & Definir & 2,80 \\
\hline & Sustituir & 0,10 \\
\hline \multirow{4}{*}{ Aplicar } & & 10,60 \\
\hline & Ejecutar & 0,10 \\
\hline & Transferir & 2,10 \\
\hline & Hacer hipótesis & 8,40 \\
\hline \multirow{6}{*}{ Analizar } & & 7,40 \\
\hline & Distinguir & 1,30 \\
\hline & Explicar & 4,50 \\
\hline & Comparar & 0,80 \\
\hline & Categorizar & 0,00 \\
\hline & Sintetizar & 0,80 \\
\hline \multirow{3}{*}{ Evaluar } & & 8,40 \\
\hline & Juzgar & 7,20 \\
\hline & Justificar & 1,20 \\
\hline \multirow{2}{*}{ Crear } & & 0,5 \\
\hline & Inventar & 0,5 \\
\hline
\end{tabular}

En relación con las subcategorías, la de reconocer - que corresponde a la categoría de recuperar- fue la más frecuente $(36,20 \%)$. De esta forma, es posible observar que dentro de la categoría recuperar, se trabaja fundamentalmente la subcategoría reconocer y las otras prácticamente no se toman en consideración en el momento de formular preguntas. En este sentido, las preguntas registradas apuntaban principalmente a recordar los hechos, personajes o situaciones de la historia leída. Por ejemplo, se identificaron preguntas como: “¿Qué le pasó a Choco?" (Educadora 56), realizada en la lec- 
ISSN $0123-1294$ | Educ.Educ. Vol. 18. No. 2 | Mayo-Agosto de 2015 | pp. 261-283.

Universidad de La Sabana | Facultad de Educación

tura del cuento "Choco encuentra una mamá", de la autora Keiko Kasza; o "¿Cuántos hijos tenía la gallina Serafina?" (Educadora 54), planteada en la lectura del cuento "La gallina Serafina" de Verónica Rossel; y “¿En qué se transformó Orlando?” (Educadora 60), formulada en el cuento "Orlando el Pulpo Valiente" de autor desconocido. El alto porcentaje de preguntas de reconocer es evidencia de cómo en la mayoría de los casos, por medio de la pregunta, se apeló simplemente a la memoria.

En el caso de las otras categorías, también fue posible observar que hay subcategorías que no se trabajan, como las de reproducir, sustituir, ejecutar, transferir, distinguir, comparar, categorizar, sintetizar, justificar e inventar. Este hecho es preocupante ya que la evidencia encontrada hasta el momento muestra que entre mayor variedad de trabajo en términos de diversas subcategorías, mayor es la posibilidad de consolidar dicha categoría.

\section{Frecuencia de las preguntas por tipo, categoría y momento del cuento}

Al cruzar por categoría la frecuencia del tipo preguntas, abiertas o cerradas, y el momento del cuento en que son elaboradas, fue posible observar que se mantiene la tendencia anterior, a saber: una mayor cantidad de preguntas después de la lectura del cuento (tabla 12). Sin embargo, hay una excepción a este patrón: las preguntas cerradas de la categoría comprender, con un 57,14\% de preguntas durante el cuento y un 42,85\% de preguntas después del cuento. Asimismo, también es posible señalar que las preguntas abiertas de la categoría aplicar presentan una distribución bastante más homogénea que las demás categorías en relación con los momentos del cuento,ya que un $27 \%$ de las preguntas se realizó antes, un $32 \%$ durante y $41 \%$ después de la lectura.

\section{Preguntas cerradas: cantidad y tipo de preguntas que las complementan}

El último objetivo del estudio estaba orientado a describir con qué frecuencia las educadoras for-
Tabla 12. Frecuencia de preguntas por categoría, tipo y momento del cuento

\begin{tabular}{|c|c|c|c|}
\hline Categoría & $\begin{array}{l}\text { Tipo de } \\
\text { pregunta }\end{array}$ & Momento & $\%$ \\
\hline \multirow{6}{*}{ Recuperar } & \multirow{3}{*}{ Abierta } & Antes & 10,2 \\
\hline & & Durante & 18,6 \\
\hline & & Después & 71,2 \\
\hline & \multirow{3}{*}{ Cerrada } & Antes & 12,19 \\
\hline & & Durante & 19,51 \\
\hline & & Después & 68,29 \\
\hline \multirow{6}{*}{ Comprender } & \multirow{3}{*}{ Abierta } & Antes & 10,44 \\
\hline & & Durante & 13,43 \\
\hline & & Después & 76,11 \\
\hline & \multirow{3}{*}{ Cerrada } & Antes & 0,0 \\
\hline & & Durante & 57,14 \\
\hline & & Después & 42,85 \\
\hline \multirow{6}{*}{ Aplicar } & \multirow{3}{*}{ Abierta } & Antes & 27,0 \\
\hline & & Durante & 32,0 \\
\hline & & Después & 41,0 \\
\hline & \multirow{3}{*}{ Cerrada } & Antes & 5,88 \\
\hline & & Durante & 13,72 \\
\hline & & Después & 80,39 \\
\hline \multirow{6}{*}{ Analizar } & \multirow{3}{*}{ Abierta } & Antes & 4,95 \\
\hline & & Durante & 17,82 \\
\hline & & Después & 77,22 \\
\hline & \multirow{3}{*}{ Cerrada } & Antes & 0,0 \\
\hline & & Durante & 20,0 \\
\hline & & Después & 80,0 \\
\hline \multirow{6}{*}{ Evaluar } & \multirow{3}{*}{ Abierta } & Antes & 2,22 \\
\hline & & Durante & 6,66 \\
\hline & & Después & 91,11 \\
\hline & \multirow{3}{*}{ Cerrada } & Antes & 1,68 \\
\hline & & Durante & 10,08 \\
\hline & & Después & 88,23 \\
\hline \multirow{6}{*}{ Crear } & \multirow{3}{*}{ Abierta } & Antes & 5,88 \\
\hline & & Durante & 17,64 \\
\hline & & Después & 76,47 \\
\hline & \multirow{3}{*}{ Cerrada } & Antes & 0,0 \\
\hline & & Durante & 0,0 \\
\hline & & Después & 100,0 \\
\hline
\end{tabular}


mulaban una segunda pregunta que complementaba una pregunta cerrada. Al realizar este análisis, fue posible observar que la mayor cantidad de preguntas cerradas elaboradas por las educadoras $(65,68 \%)$ no fueron seguidas por otra pregunta que las complementara (tabla 13). Este hecho se considera como algo desfavorable para el desarrollo del pensamiento de los niños, ya que las preguntas cerradas son simples y requieren de respuestas muy cortas (Monfort y Juárez, 2008), que no llevan al niño a una mayor reflexión.

Este hecho se puede apreciar en la siguiente pregunta cerrada: "¿Y qué hizo don Chancho, se puso la melena o no?" (Educadora 58), planteada en la lectura del cuento "El día de campo de Don Chancho", de la autora Keiko Kasza. En esta pregunta se limita la reflexión, ya que la respuesta está contenida en la misma. De esta forma, no obstante que las preguntas cerradas son las menos frecuentes durante la hora del cuento (23,65\%), es preocupante que la mayoría de ellas no esté seguida por otras preguntas que les permitan a los estudiantes alcanzar mayores niveles de elaboración y de procesamiento cognitivo.

Tabla 13. Preguntas cerradas que son seguidas por una complementaria

\begin{tabular}{|c|c|}
\hline $\begin{array}{c}\text { Seguidas por una pregunta } \\
\text { complementaria }\end{array}$ & $\%$ \\
\hline No & 65,68 \\
\hline Sí & 34,31 \\
\hline Total & 100 \\
\hline
\end{tabular}

Adicionalmente, se analizó si las preguntas complementarias a las preguntas cerradas eran de tipo abierto o cerrado, observándose un aumento en este último tipo de pregunta comparado con el momento cuando estas fueron formuladas por primera vez. De esta forma, las preguntas cerradas aumentaron de un 23,65\% cuando se formulaban por primera vez (tabla 8 ), a un $37,63 \%$ cuando estas se elaboraban como complementarias a una primera pregunta cerrada (tabla 14). En relación con este punto, es importante mencionar que se hubiera esperado que las preguntas complementarias fueran casi en su totalidad abiertas, ya que persiguen que el niño piense y procese de manera más elaborada sus respuestas. Sin embargo, esto no fue así en aproximadamente una de cada tres preguntas complementarias, como es el caso de la pregunta "¿Él estaba contento con su peso?", que formuló la Educadora 46, luego de haber preguntado a los estudiantes: "¿El niño quiso ser gordo?”, en el contexto de la lectura del cuento "Soy un niño", de José Luis García Sánchez.

Tabla 14. Frecuencia de preguntas complementarias por tipo

\begin{tabular}{|c|c|}
\hline Tipo de Pregunta & $\%$ \\
\hline Abierta & 62,36 \\
\hline Cerrada & 37,63 \\
\hline Total & 100 \\
\hline
\end{tabular}

A pesar de que el número de preguntas cerradas fue más alto cuando fueron preguntas complementarias que cuando fueron formuladas por primera vez, es posible rescatar que del total de las preguntas complementarias, el 62,36\% correspondieron a preguntas abiertas y un $37,63 \%$ a preguntas cerradas, lo que permite afirmar que la mayoría de las preguntas complementarias fueron abiertas, promoviendo de esta forma una mayor elaboración de las respuestas dadas por los niños.

Finalmente, se analizó a qué categoría correspondían las preguntas complementarias, observándose que las tres categorías más frecuentes eran las de evaluar, aplicar y recuperar (tabla 15). De esta forma, es posible señalar que, en términos generales, las preguntas complementarias demandan mayores niveles de procesamiento cognitivo que las primeras, ya que un $47,31 \%$ de ellas correspondían a la categoría evaluar, que es uno de los niveles más altos identificados por la taxonomía utilizada, solo sobrepasado por la categoría crear.

Se considera que esta tendencia respondería a la necesidad de las educadoras de indagar en las 
respuestas que expresan sus alumnos y favorecer el desarrollo del pensamiento por medio de la argumentación, la valoración y la aplicación a la propia experiencia. Por ejemplo, en la lectura del cuento "Mi mamá es preciosa", de la autora Carmen García Iglesias, la educadora 46 pregunta: "¿Cruzan la calle solos?", y luego complementa con la siguiente pregunta perteneciente a la categoría Evaluar: “Está bien que crucen la calle solos?” En la lectura del cuento "Mi día de suerte", de la autora Keiko Kasza, la educadora 47 pregunta: "¿Habrá terminado el cuento ahí?", y luego complementa con la siguiente pregunta de la categoría Aplicar: “¿Qué habrá pasado después con el oso?"

Tabla 15. Frecuencia de preguntas complementarias por categoría

\begin{tabular}{|c|c|}
\hline Categoria & \% \\
\hline Recuperar & 13,97 \\
\hline Comprender & 7,52 \\
\hline Aplicar & 21,50 \\
\hline Analizar & 7,52 \\
\hline Evaluar & 47,31 \\
\hline Crear & 2,15 \\
\hline Total & 100 \\
\hline
\end{tabular}

\section{Conclusiones}

Aunque distintos autores en la literatura internacional han señalado la importancia de que los educadores realicen preguntas abiertas (de Rivera et al., 2005; Monfort y Juárez, 2008), ya que ellas promueven mayores grados de reflexión y respuestas más complejas tanto cognitiva como lingüísticamente, los resultados de este estudio sugieren que, para promover altos niveles de procesamiento cognitivo en los estudiantes, el realizar preguntas de este tipo no es suficiente. Por el contrario, fue posible observar que a pesar de que la mayoría de las preguntas realizadas por las educadoras de segundo nivel de transición durante la hora del cuento son abiertas, el nivel cognitivo que estas demandan es notablemente bajo. Este hecho se debe a que las preguntas correspondían mayoritariamente a la categoría de recuperar, lo que significa que estaban orientadas principalmente a que los estudiantes identificaran y recordaran aspectos tratados en el texto, sin promover mayores niveles de elaboración al respecto.

Asimismo, llama la atención no solo el bajo nivel de frecuencia de las preguntas de mayor complejidad, sino también el hecho de que la mayor cantidad de preguntas se hayan elaborado después del cuento, en desmedro de aquellas realizadas antes y durante su lectura. Cada momento de la hora del cuento tiene un potencial particular para promover en los estudiantes distintos niveles de procesamiento cognitivo. Antes de la lectura es la instancia propicia para que estos activen sus conocimientos previos y desarrollen pensamiento hipotético a través de pensar en escenarios posibles. Es por ello que preguntas que inviten a realizar inferencias, a relacionar creencias o significados con imágenes o títulos, es una oportunidad que está siendo subvalorada en este contexto.

Del mismo modo, durante la lectura las educadoras deberían promover procesos de pensamiento de tipo predictivo e inferencial, ya que los estudiantes cuentan con suficiente información sobre el cuento para pronosticar sucesos probables de acuerdo con el desarrollo de la historia. Además de ello, durante la lectura del cuento es posible desplegar una infinidad de preguntas que posibilitan el desarrollo del pensamiento. Interrogantes que apunten a la distinción, comparación, categorización, por nombrar algunos, están escasamente presentes en la intención implícita de las preguntas formuladas, y son necesarias para robustecer el pensamiento. Finalmente, después del cuento los estudiantes debieran ser invitados a realizar procesos de sintesis, metacognición y transferencia (Castro y Rodríguez, 2005; Ibarra, 2000). Sin embargo, a lo largo del estudio dichos procesos superiores de pensamiento estuvieron prácticamente ausentes. 
Los datos de este estudio sugieren que una de las formas de elevar los niveles cognitivos demandados por las preguntas y, de esta forma, promover los procesos de pensamiento señalados es a través de una mejor y mayor utilización de preguntas complementarias. Aunque en la mayoría de los casos las educadoras no formulaban una segunda pregunta que complementara una pregunta cerrada, cuando sí lo hacían, el nivel de procesamiento demandado por ellas era muchísimo más alto. De esta forma, aunque en términos generales las preguntas cerradas son simples y requieren de respuestas extremadamente cortas que no llevan a los estudiantes a mayores grados de reflexión (de Rivera et al., 2005; Monfort y Juárez, 2008; Polanco, 2004), cuando estas son acompañadas por otras preguntas abiertas promueven niveles de elaboración más altos de parte de los estudiantes. Esta evidencia parece sugerir que, más allá de si la pregunta original es abierta o cerrada, el hacer varias preguntas complementarias abiertas a una pregunta original promueve que los estudiantes profundicen en sus reflexiones $y$, por tanto, vayan aumentando los niveles de complejidad de sus respuestas y de los procesos de elaboración asociados a ellas. Así, más allá de la frecuencia de las preguntas abiertas, cerradas o complementarias, lo importante es advertir qué tipo de habilidades de pensamiento se están promoviendo escasamente en la interacción profesor-alumno (por ejemplo, reproducir, sustituir, ejecutar, transferir, distinguir, comparar, categorizar, sintetizar, justificar e inventar, etc.).

\section{Limitaciones y contribuciones del estudio, y próximas líneas de investigación}

Las principales limitaciones de este estudio se relacionan con las características y la diversidad de la muestra. En primer lugar, es posible señalar que el hecho de seleccionar comunas que tuvieran similitudes en términos de sus indicadores económicos, educativos y sociales fue deliberado para poder reunir a todas las comunas participantes en un solo grupo, esto presenta limitaciones en términos de la representatividad de la muestra y la posibilidad de generalizar los resultados a otros contextos educativos. Esta limitación se intensifica por el hecho de que todos los establecimientos participantes eran de dependencia particular subvencionada, de manera que nuevamente es posible pensar que los resultados podrían ser transferibles solo a este tipo de establecimientos, más allá de que ellos representan la mayor matrícula a nivel nacional.

Adicionalmente, al interior de la muestra cada comuna tiene una representatividad bastante dispar en términos de la cantidad de establecimientos incluidos, ya que estos varían desde 1, como es el caso de la comuna de Providencia, a 11 en el caso de San Joaquín, repercutiendo en el grupo de educadoras de párvulos incluido por cada comuna. Esta limitación encuentra su justificación no solo en el hecho de que la cantidad de establecimientos particulares subvencionados en cada comuna era diversa, sino también en el número de los que aceptaron la invitación a participar en el estudio. De esta forma, esta era una variable que escapaba a la voluntad y capacidad de planificar del equipo de investigación.

Más allá de lo anterior, es posible señalar que los resultados del estudio son relevantes al aportar evidencia empírica del tipo de preguntas que las educadoras de párvulos realizan a sus estudiantes de segundo nivel de transición, y del nivel de demanda cognitiva que estas implican, siendo pionero en esta línea no solo a nivel nacional sino también latinoamericano. De esta forma, a pesar de toda la literatura existente que demuestra la importancia que las preguntas de calidad tienen en la promoción de procesos cognitivos superiores, la evidencia a este respecto es bastante limitada. Del mismo modo, las implicancias que el estudio tiene son relevantes ya que permiten retroalimentar los procesos de formación inicial y profesional docente de manera que los educadores valoren la importancia de formular preguntas de calidad y desarrollen las habilidades para construirlas, evaluarlas y utilizarlas como un verdadero instrumento pedagógico y de aprendizaje. 
Para finalizar, aunque los resultados discutidos aportan evidencia relevante y atinente a la problemática educativa actual, abren nuevas interrogantes que necesitan ser respondidas para desarrollar una mejor y más profunda comprensión de los factores que se encuentran a la base de estos procesos, facilitando o entorpeciendo la utilización de la pregunta como recurso de aprendizaje. De esta forma, aún es necesario conocer cuáles son las variables que explican la elaboración de una pregunta pedagógica de calidad, qué rol juegan las preguntas complementarias en este proceso, cómo se están formando los educadores para lograrlo, qué papel juega el tipo de texto que se utiliza en el tipo de preguntas formuladas, y si existen diferencias entre escuelas con estudiantes de distintos niveles de capital cultural, entre otros.

\section{Agradecimientos}

Nuestro más sincero agradecimiento a todas las educadoras de párvulos que con su generosa y desinteresada participación hicieron posible la realización de este estudio. Asimismo, nos gustaría agradecer a nuestras estudiantes Rosario Álvarez, Rosario Benoit, Constanza Caruso, María Jesús Donoso, Francisca Gatica, Pauline Gigoux, Valentina Gómez, Rosario Hoppe, Valentina Infante, Blanca Irarrázaval, María Cecilia Velasco y Catalina Winkler, quienes fueron una pieza fundamental en todo el proceso de realización de este proyecto de investigación, especialmente en la recolección y el análisis de los datos.

\section{Referencias}

Abarca, J., Sala, M., y Marzo, L. (2002). La educación emocional y la interacción profesor/a-alumno/a. Revista electrónica interuniversitaria de formación del profesorado, 5 (3), 1.

Álvarez, E. (2010). Creatividad y pensamiento divergente. Desafío de la mente o desafío del ambiente. Revista Interact, 1-28.

Anderson, L.y Krathwohl, D. (2001). A taxonomy for learning, teaching and assessing: A revision of Bloom's taxonomy of educational objectives. United States: Pearson Education.

Arancibia, V., Herrera, P.y Strasser, K. (2011). Manual de Psicología Educacional. Santiago de Chile: Ediciones Universidad Católica de Chile.

Banyard, P., Cassells, A., Green, P., Hartland, J., Hayes, N. y Reddy, P. (1995). Introducción a los procesos cognitivos. Barcelona: Ariel.

Bermeosolo, J. (2004). Psicología del Lenguaje. Santiago: Ediciones Universidad Católica de Chile.

Berríos, A., Bustos, C., Torres, C., Osorio, A., Oyaneder, M., Merino et al. (2010). Plan Nacional de Fomento de la Lectura. Guía para las Educadoras de Párvulos de los Niveles de Transición. Chile: Unidad de Educación Parvularia, Plan Nacional de Fomento de la Lectura, División de Educación General, Ministerio de Educación, Gobierno de Chile.

Beuchat, C., Munita, F., Riquelme, E., Chambers, A., Patte, G., Trelease, J. y Edwards, A. (2013). A viva voz. Lectura en voz alta. Chile: Bibliotecas Escolares CRA, Unidad de Currículum y Evaluación, Ministerio de Educación, Gobierno de Chile, Santiago. 
Bloom, B. et al. (1990). Taxonomía de los objetivos de la educación: la clasificación de las metas educacionales. Buenos Aires: El Ateneo.

Burton, L. y Habenicht, D. J. (2004). Lograr que los alumnos piensen. Uso de preguntas de modo efectivo en la sala de clases. Revista de Educación Adventista, 19,17-21.

Candelas, M. (2011). Sobre las preguntas infantiles y su relevancia para el cambio educativo. Escuela abierta, $14,111-122$.

Childs, A. y Mcnicholl, J. (2007). Investigating the Relationship between Subject Content Knowledge and Pedagogical Practice through the Analysis of Classroom Discourse. International Journal of Science Education, $13,1629-1653$.

Cohen, L., Manion, L. y Morrison, K. (2007). Research Methods in Education. Oxford: Routledge Publishers.

Collante, C. (2010). El valor de la pregunta. Revista Investigación en Marcha, 3, 21-27.

Craig, G. (2001). Desarrollo Psicológico. México: Pearson Educación.

Davidoff, L. (1990). Introducción a la psicología. Barcelona: McGraw-Hill.

De Rivera, C., Girolametto, L., Greenberg, J. y Weitzman, E. (2005). Children's responses to educators' questions in day care play groups. American Journal of Speech-Language Pathology, 14, 14-26.

Dewalt, K. M. y Dewalt, B. R. (2002). Participant observation: a guide for fieldworkers. Walnut Creek, CA: AltaMira Press.

Dickinson, D. K. y Smith, M. W. (1994). Long-term effects of preschool teachers' book readings on lowincome children's vocabulary and story understanding. Reading Research Quarterly, 29 (2), 105-122.

Doyle, W.y Carter, K. (1996). Educational Psy chology and the Education of Teachers: A reaction. Educational Psychologist, 31, 51-62.

Edwards, A. (2008). Hora del cuento. Chile: Editorial Universitaria.

Eyzaguirre, B. y Fontaine, L. (2008). Diferencias de aprendizaje y la enseñanza de la lectura. En Parra, D. (ed.). Las escuelas que tenemos (pp. 173). Santiago de Chile: Centro de Estudios Públicos.

Feldman, R. (2007). Desarrollo psicológico. México: Pearson Educación.

González García, J. (2007). Debates en el aula preescolar a partir de cuentos: Estudio comparado en España y México. Perfiles educativos, 29 (118), 54-78.

González, R., Preiss, D. D. y San Martín, E. (2008). Evaluando el discurso docente: desarrollo de un modelo de Rasch a partir de la evidencia audiovisual de profesores chilenos de primer ciclo de educación básica en el Área de Lenguaje. Revista Iberoamericana de Evaluación Educativa, 2, 137-147.

Hernández, R., Fernández, C. y Baptista, P. (2010). Metodología de la investigación. México: McGraw-Hill. 
ISSN $0123-1294$ | Educ.Educ. Vol. 18. No. 2 | Mayo-Agosto de 2015 | pp. 261-283.

Universidad de La Sabana | Facultad de Educación

Hiebert, J., Gallimore, R., Garnier, H., Givvin, K. B., Hollingsworth, H.y Jacobs, J. (2003). Teaching Mathematics in Seven Countries: Results from the TIMSS 1999 Video Study. Washington DC: U.S. Government Printing Office.

Holt-Reynolds, D. (1992). Personal History-based Beliefs as Relevant Prior Knowledge in Course Work. American Educational Research Journal, 44, 279-287.

Honig, A. S. y Wittmer, D. S. (1982). Teacher questions to male and female toddlers. Early Child Development and Care, 9, 19-32.

Huck, C. (1976). Children's Literature in the Elementary School, 3 ed. United States: Holt.

Ibarra, M. G. (2000). Estrategias antes, durante y después de la lectura. México: Portal Académico IEMS. Recuperado el 12 de enero de 2014 de http://academicos.iems.edu.mx/cired/docs/tg/ColoquiolnstitucionalPo e/6Estrategias_antes_durante_despues_de_la_lectura_Guadalupelbarra.pdf

Instituto Nacional de Estadísticas, Gobierno de Chile (2011). Compendio Estadístico, Estadísticas de Educación, Cultura y Medios de Comunicación. Santiago de Chile: Instituto Nacional de Estadísticas.

Justice, L. y Pentimonti, J. (2010). Teachers' use of scaffolding strategies during read alouds in the preschool classroom. Early Childhood Education Journal, 37 (4), 241-248.

Kagan, D. (1992). Implications of Research on Teacher Belief. Educational Psychologist, 27, 65-90.

Mcloughlina, D. y Mynard, J. (2009). An analysis of higher order thinking in online discussions. Innovations in Education and Teaching International, 46, 147-160.

Monfort, M. y Juárez, A. (2008). El niño que habla: el lenguaje oral en el preescolar. Madrid: CEPE.

Moses, A. (2005). Text Talk: A Summary of Research. New York: Scholastic Inc. Research and Results.

Muñoz, B. y Anwandter, A. (2011). Manual de lectura temprana compartida. ¿Por qué es importante y cómo leer con niños y niñas de o a 7 años? Chile: Consejo Nacional de la Cultura y las Artes, Fondo Nacional de Fomento del Libro y la Lectura, Gobierno de Chile.

Navarro, E. B. (2000). Alfabetización emergente y meta cognición. Revista Signos, 33.

Otto, B. (2008). Literacy development in early childhood: Reflective teaching for birth to age eight. USA: Prentice Hall.

Papalia, E. D., Wendkos, S. y Duskin, R. (2009). Desarrollo cognitivo en la segunda infancia. En Olivares, S.y Padilla, G. (trad.). Psicología del desarrollo. México: McGraw-Hill.

Polanco, A. (2004). La pregunta pedagógica en el nivel inicial. Revista electrónica Actualidades Investigativas en Educación, 4 (2). Recuperado el 12 de enero de 2014 de www.eduteka.org.

Rodari, G. (2008). Gramática de la fantasía: introducción al arte de inventar historias. Buenos Aires: Colihue.

Rodríguez, C. (2007). Componentes y estrategias de comprensión lectora en educación primaria. AjuscoMéxico: Universidad Pedagógica Nacional, Unidad Ajusco. 
Salazar, J. y Marqués, M. D. L. L. (2012). Acompañamiento al aula: una estrategia para la mejora del trabajo pedagógico. RIEE. Revista Iberoamericana de Evaluación Educativa, 5 (1), 10-20.

Smith, F., Hardman, F., Wall, K. y Mroz, M. (2004). Interactive whole class teaching in the National Literacy and Numercy Strategies. British Educational Research Journal, 30, 395-411.

Swartz, S. (2010). Cada niño un lector: estrategias innovadoras para enseñar a leer y escribir. Santiago de Chile: Ediciones UC.

Thuel, L. (1995). La pregunta pedagógica: un medio para desarrollar el pensamiento. En Memoria // Simposio Educación Preescolar. San José: Universidad de Costa Rica.

Vigotsky, L. (1986). Thought and language. Cambridge: MIT.

Villén, S. J. (2009). Estrategias para desarrollar la capacidad creativa del alumnado. Revista electrónica Innovación y Experiencias Educativas, 22, 1-8.

Wasik, B. (2010). What teachers can do to promote preschoolers' vocabulary development: strategies from an effective language and literacy professional development coaching model. The Reading Teacher, 63, 621-633.

Wells, G. (2002). The Role of Dialogue in Activity Theory. Mind, Culture and Activity, 9, 43-66.

Wiseman, A. (2011). Interactive read alouds: Teachers and students constructing knowledge and literacy together. Early Childhood Education Journal, 38 (6), 431-438.

Zucker, T. A., Justice, L. M., Piasta, S. B. y Kaderavek, J. N. (2010). Preschool teachers' literal and inferential questions and children's responses during whole-class shared reading. Early Childhood Research Quarterly, 25 $(1), 65-83$.

Zuleta Araújo, O. (2005). La pedagogía de la pregunta, una contribución para el aprendizaje. Revista electrónica Educere, 9 (28), 115-119. 
